\title{
Image Scaling Using Transform Coding with Quad tree and Huffmann Coding
}

\author{
Swathi $\mathrm{J}^{1^{*}}$, Deepika Sasi ${ }^{2}$, Aleena Joseph ${ }^{3}$, Akash Vijayan ${ }^{4}$, Ashitha $\mathbf{P}^{5}$ \\ ${ }_{1,2,3,4,5}$ M. Tech Student, APJ Abdul Kalam Technological University, ECE Department, \\ TKM College of Engineering, Karikode, Kollam, Kerala, India. *swathiacer@gmail.com
}

\begin{abstract}
With the growth of the business sector for digital media resources, for example computerized images and videos, the analysis of pressure methods has evolved. The creation of better quality and less expensive picture handling innovations has added to a continuous development in both picture size and goals, adding to a more prominent impact on the formation of compelling pressure frameworks. Photograph pressure is additionally one of the basic purposes behind the putting away or transmitting of information by means of the contact media. This paper recommended a cutting edge refreshed technique to expand the pressure proportion, the PSNR esteems and the decrease of encoding time. Utilizing change coding with quad tree decay and Huffman coding to authorize picture pressure. Utilizing the limit estimation of 0.2 and Huffman coding for the translating and encoding of picture systems, for example, these, picture pressure was executed. Pinnacle Signal to Noise Ratio and pressure proportion are determined for three kinds of pictures. The trial of the Mat lab investigation show that the Quad tree deterioration technique exhibits point by point change in pressure proportions and PSNR esteems.
\end{abstract}

Keywords - Quadtree, Huffmann encoding, decoding, image compression, PSNR

\section{INTRODUCTION}

Speaking to the realistic portrayal of somebody requires a lot of bytes for information. By lessening the size of the record, we utilize the procedures of picture pressure without trading off the nature of the picture. The compacted picture is spoken to by less bits than the underlying one. The key reason for picture pressure is to lessen the multifaceted nature and absence of significance of the document, permitting it simpler to store and transmit pictures proficiently. Pressure is intended to lessen the volume of material used to depict a book, picture or video without diminishing the precision of the first information. Picture pressure can be what might be compared to picture pressure. As an outcome, the ideal stockpiling limit is constrained; as a result, more photos can be handled and transmitted all the more proficiently to ration time by moving data transfer capacity.

Coding excess is available where the right code words allotted to the predetermined occasions have not been decided to assess the likelihood of the occasions bringing about coding repetition. For picture pressure, redundancies are separated into three sorts: repetition naming, between pixel excess and psycho-visual excess. Entomb pixel repetition because of the comparability between the pixels of the picture. Because of subtleties missing from the Human Sensory System, truly insignificant information is named psycho-visual repetition. We may acquire a recreated picture from the packed pictures. This technique is alluded to as the converse or decompression cycle.

\section{RELATED WORK}

Chowdhury et al. [1] proposed picture pressure using a safe wavelet change. Shen et al. [2] investigated the procedure of versatile picture pressure subject to vector quantization. Sahami et al. [3] introduced a bi-level picture encoding procedure using neural systems. Lossy pressure suggests misfortune; compacted picture is equivalent to the underlying uncompressed picture yet not equivalent to the past one as the pressure procedure. Tan et al [4] put forward a picture encoding methodology using a limit esteem calculation reference level.

\section{METHODOLOGY}

\subsection{Image-compression recognition methods}

We are using Transform coding, Quardtree decomposition and Huffmann coding for the image compression.

\subsubsection{Coding Convert}

Convert coding is utilized to decipher pixel esteems for the spatial picture to change esteems for coefficient. Since this is a straight technique so no data is lost, the measure of produced coefficients is equal to the quantity of changed pixels. A pixel's vitality can be portrayed as the square of 
any scaling factor times its size. Additionally, any scaling variable might be depicted as the vitality of a change coefficient as the square of its worth occasions. The combined vitality of the pixels in a picture will at present be equal to the absolute vitality in the coefficients of interpretation for the right scaling factor.

\subsubsection{Decomposition of Quadtree}

In the decay of the quad tree, each picture is isolated into four quadrants characterized as the area. At that point, another area is part into 4 quadrants known as extents, and to discover their match, each range is contrasted with spaces. With the guide of entropy we discover play. Each square of range looks for its match in the event that it has not discovered its match, at that point the square of range is part into 4 quadrants before it looks for coordinate. This procedure is reproduced until it gets its counterpart for all range square.

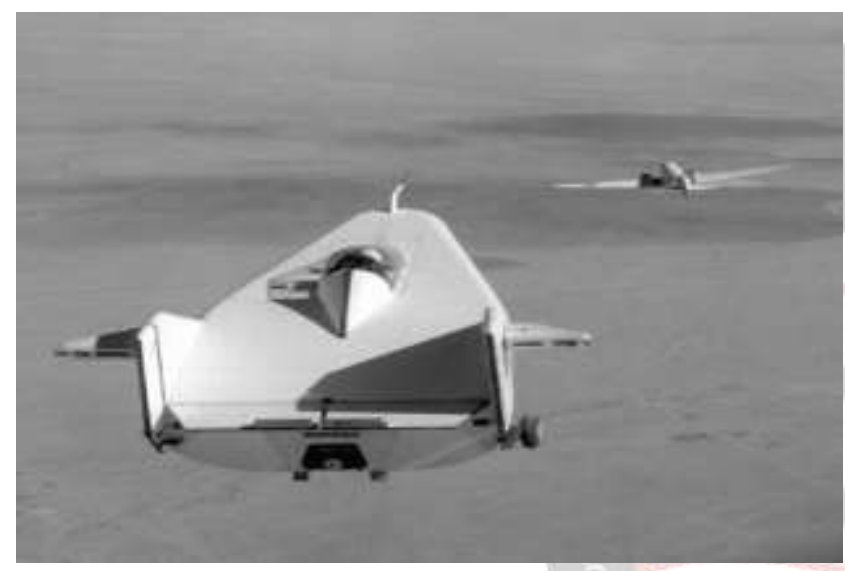

Fig 1: Initial Data

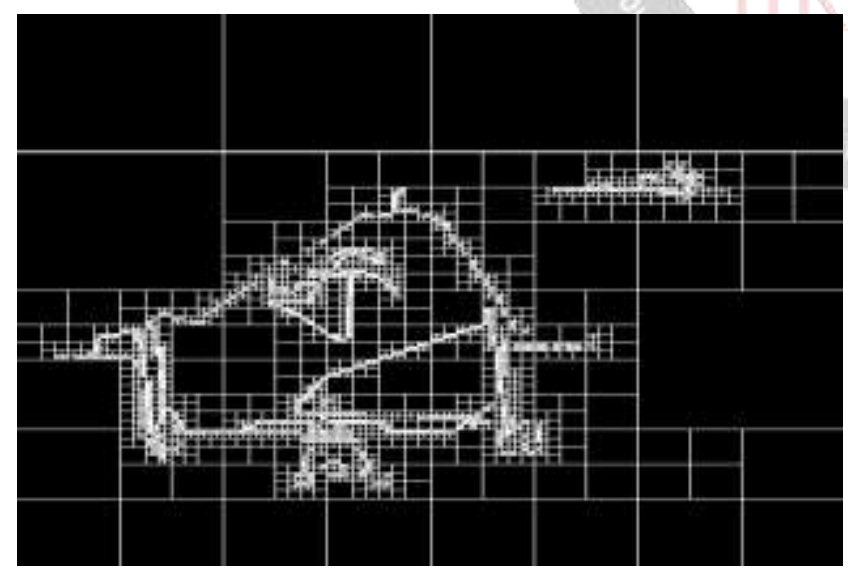

Fig 2: Decomposition of Quadtree

\subsubsection{Coding with Huffmann}

Huffman coding is proficient entropy encoding for lossless pressure of pictures. Huffman coding is an improved prefix innovation utilized for encoding of lossless outcomes. David A. Huffman had constructed a calculation to find a document this way. This calculation is a sort of numerical coding which functions admirably when sending content which faxes. It is a lossless calculation for the pressure of records. The essential standard behind this calculation is to distribute input characters with the variable length codes. The length of the code apportioned relies upon the comparing character frequencies.

The most normally showing up characters were given restricted code. Bigger coding is distributed to the character which happens least often. There are two key strides of Huffman, one of which is to take the info character and make a Huffman tree from it, and the other is to allot codes to specific characters through the Huffmantree.

Partition the underlying limit disintegration picture utilizing Quadtree is 0.2 , least measurements and normal measurements are 2 and 64. Record in Quadtree decomposition the estimations of $\mathrm{x}$ and $\mathrm{y}$ organizes, mean worth, and square measurement. To finish the encoding of the document, record the fractal coding subtleties utilizing Huffman coding and measure the pressure proportion. For the encoding picture which unravels Huffman to reproduce the image and to quantify PSNR.

\section{SYSTEM OVERVIEW}

The measures to the machine calculation are as per the following.

Utilizing change code, the information picture will be packed. The changed picture is then to be isolated by utilizing the limit estimation of Quad tree deterioration as 0.2 , the base and greatest measurements are 2 and 64, individually. Encoding time, pressure proportion and peak sign to commotion proportion are the last advance. To finish the encoding we use Huffman coding and Huffman interpreting to reproduce the record.

Read picture information.

Split the image into $8 X 8$ segments.

Add DCT to 8X8 segments separately.

Apply quad tree decay to DCT changed picture isolated by quad tree deterioration where 0.2 is the limit esteem. Record square size is importance from decay of quad tree.

Use coding for Huffman. At input we take an arrangement of explicit character alongside their recurrence events and this Huffman tree yield.

Measure the measure of pressure.

Decoding Huffman.

Photo decompressed.

Determine PSNR giving the proportion of consistency figuring between the underlying picture and the compacted picture. PSNR qualities ought to be solid. 


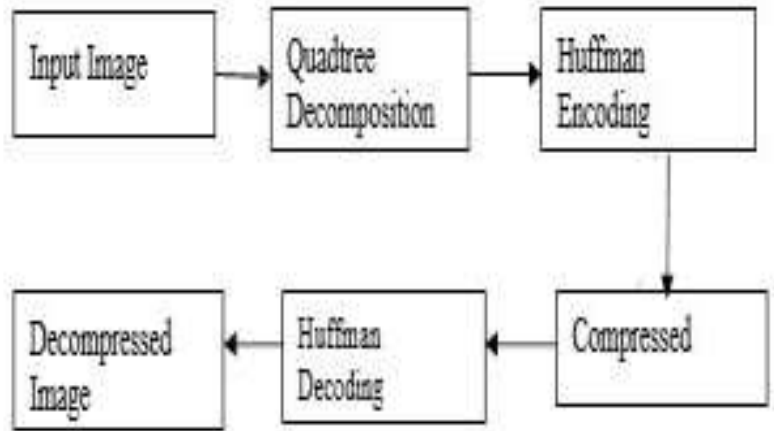

Fig 3: System Architecture

\section{RESULT AND DISCUSSIONS}

The algorithm was rendered in Matlab to code and decode Lena's satellite picture of size 512 X 512. The trigger value is 0.2 , the limit is 64 and the minimum is 2 . After Quadtree decomposition, Huffman encoding and decoding, the initial image and the decompression picture are seen in Figure8.

Following pressure the reestablished ancient rarities are found in Figures 6, 7 and 8. For the Numbers, the reestablished picture quality is obviously fine, as the PSNR esteems for the photographs propose marginally higher qualities greater than 28 . The statistics emphatically show that the Quadtree deterioration pressure looks for effective applications to get huge pressure proportions with moderately not too bad PSNR values.

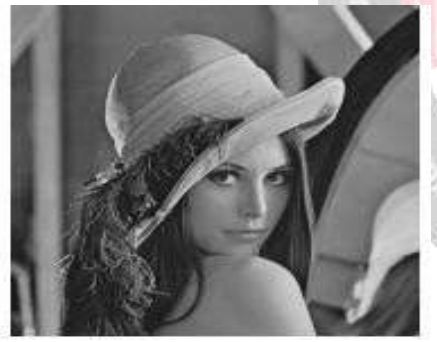

Fig 4: Initial Image /Lena Image

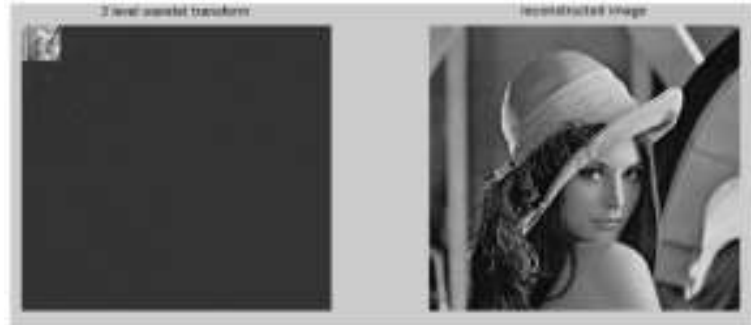

Figure 5: Compression of the picture by wavelet transformation

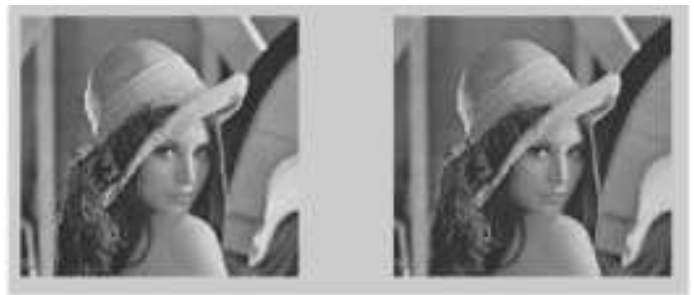

Figure 6: DCT Zonal efficiency: Efficiency level

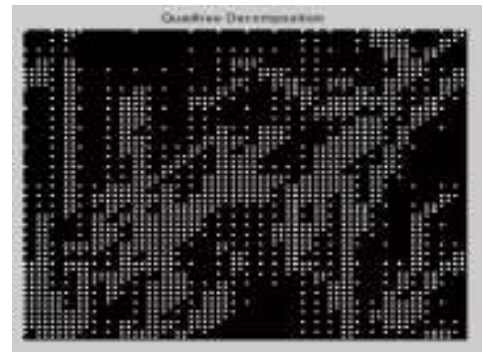

Figure 7: Decomposition of Quadtree

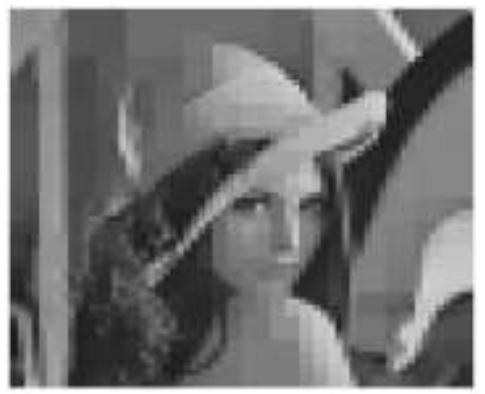

Figure 8: Decompressed image

\section{CONCLUSIONS}

In this paper the use of change coding with quadtree disintegration and Huffman coding is recommended as a pressure calculation. The method packed the pictures adequately, and discovered the ideal PSNR. Since in our proposed arrangement we are not concentrating on noncovered articles or sub-pictures, so here things have been excluded by working all in image instead of parts. Numerous present methods produce ancient rarities that impede. From the reenactment tests, we have demonstrated that the proposed philosophy requests less execution time and is powerful in compacting pictures of different structures. We additionally worked with a solitary component, as it needs less execution time as a result of another advantage. Numerous discrete fragmentary cosine changes for picture pressure ought to be presented in future region.

\section{REFERENCES}

[1] M. MozammelHoque Chowdhury, Amina Khatun, "Image Compression Using Discrete Wavelet Transform", International Journal of Computer Science Issues, Vol. 9, Issue 4, No 1, July2012.

[2] Jau-JiShen and Hsiu-Chuan Huang, "An Adaptive Image Compression Method Based on Vector Quantization”,"IIEEE, pp. 377-381,2010.

[3] S. Sahami and M.G. Shayesteh, "Bi-level image compression technique using neural networks", IETImage Process, Vol. 6, Iss. 5, pp. 496-506,2012.

[4] Yi-Fei Tan and Wooi-Nee Tan, Image Compression Technique Utilizing Reference Points Coding with Threshold Values,IEEE, pp. 74-77,2012. 
[5] Rafael C. Gonzalez, Richard E. Woods. Digital Image Processing, 2nd ed., 1992, NJ: Prentice Hall.

[6] FirasA. Jassim and Hind E. Qassim, "Five Modulus Method for Image Compression”, SIPIJ Vol.3, No.5, pp. 19-28,2012.

[7] Fisher Y, editor (1995) "Fractal image compression: theory and application", New York, Springer- Verlag.

[8] Arnaud E. Jacquin, (1993) "Fractal image coding”, Proceedings of IEEE VOL.81, pp.14511465

[9] Bohong Liu and Yung Yan, (2010) "An Improved Fractal Image Coding Based on the Quadtree", IEEE 3rd International Congress on Image and Signal Processing, pp. 529-532.

[10] Dr. Muhammad Kamran, Amna Irshad Sipra and Muhammd Nadeem, (2010) "A novel domain Optimization technique in fractal image compression", IEEE Proceedings of the 8th World Congress On Intelligent Control and Automation, pp. 994-999. 